SLAC-PUB-12849

October 2007

\title{
On "DARK ENERGY FROM ANTIMATTER by Walter R. Lamb"
}

\author{
H. Pierre Noyes * \\ Stanford Linear Accelerator Center \\ Stanford University, Stanford, CA 94309
}

This paper is written in the context of the following manuscript ${ }^{\dagger}$

\footnotetext{
*Work supported in part by Department of Energy contract DE-AC02-76SF00515.

$\dagger$ The manuscript reproduced here — except for minor proof corrections — was received by Physics Essays on 6 June 2006. Unfortunately Walter R. Lamb(WRL)'s illness and death prevented completion of the normal publication process. H.Pierre Noyes (HPN) had an informal agreement with WRL to see the manuscript through press. The somewhat unusual way in which this is being accomplished is occasioned by the fact that HPN had decided, prior to WRL's illness, not to offer his services as a referee because he felt that his long friendship with WRL and detailed discussion of these ideas with him over the years would prevent any such refereeing from being unbiased. However HPN assures the reader that (a) HPN felt and continues to feel that WRL's ideas deserve a hearing; (b) that HPN believes that WRL would have wanted his manuscript published in the form which follows; and (c) that WRL would have persisted in that wish despite the somewhat critical tone of some of the discussion of WRL's ideas which is presented by HPN following the text of WRL's paper. This paper is expected to appear in the upcoming (March 2008) issue of Physics Essays dedicated to the memory of Dr. V. Arunasalam.
} 


\title{
DARK ENERGY FROM ANTIMATTER \\ Walter R. Lamb
}

\begin{abstract}
It is found that dark energy consists of gravitationally repulsive antimatter. The implications are discussed. A solar system proof is provided.
\end{abstract}

Key Words: Dark Energy, Anti-matter, Repulsive Gravity

It is proposed that the cosmological dark energy consists primarily of anti-particle anti-gravity. According to the theory of Noyes and Starson in their paper "Discrete Antigravity" [1] "anti-particles near the surface of the earth will 'fall' up with the same acceleration that the corresponding particles fall down". Koberlein[2] analyzed this crossing symmetry in the mainstream Physics context and found only an apparent violation of the weak equivalence principle, which he handles by modifying gravitational theory to agree with both general relativity and the Noyes prediction. There have been other speculations on the gravitational properties of anti-particles; only two examples are cited here: [3] and [4]. The discussion herein assumes with Wheeler[5] that "We now know that for every particle in nature there is an anti-particle" and with Hoyle, Burbridge, and Narliker[6] "as well as the distinction between the universal sea of particles and a sea of separated fireballs there is the difference that in the big-bang case the sea is a balanced particle-antiparticle system, whereas the fireballs are of particles only ...". The big-bang requires a major, cosmological separation of matter and antimatter or annihilation. The difficulty of handling this is analyzed in a recent paper[7] where the possibility of small amounts of antimater in domains surrounded by matter is only entertained for the early universe.

Extending the Noyes theory, I propose that antiparticles are gravitationally selfrepulsive, - no collective or cluster masses such as molecules, pebbles, stars and galaxies occurring. Early nucleosynthesis may have formed antiparticle atoms, which would likewise be gravitationally repulsive. Antimatter would be mostly exterior to visible galaxies and possibly, excluding major matter events such as a supernova,

\footnotetext{
${ }^{\ddagger}$ deceased
} 
would be between galaxies and pushing them both apart and individually compact as observed.

In that case the Universe would look like a sponge, as indeed the latest Hubble pictures show[8], with intergalactic voids composed of antiparticles as a virtually transparent gas and the gravitationally bound portions composed of the molecules, planets, stars, and galaxies that we observe. One can then expect that the volume of 'void' should greatly exceed the volume of visible matter, which is observationally true.

This approach and understanding solves all or nearly all the dark energy problem. At least half of the coherence of galaxies may be provided by the repulsive gravitational force between the galaxies. The curvature of space is characterized by the density parameter $\Omega_{\text {total }}=1$ which was determined from the Boomerang experiment $[9]$ to be equal to 1 which implies a flat space. The Boomerang measurements coupled with the data from Type Ia supernova studies also provide $\Omega_{\Lambda}$ (vacuum energy density) $=$ 0.7 , and $\Omega_{M}$ (matter density) $=0.3$ where $\Omega_{\text {total }}=\Omega_{\Lambda}+\Omega_{M}$. Here the term vacuum energy density may be about half replaced by anti-particle dark energy, $\Omega_{A P}$. The 0.7 value is close to double the 0.3 value which is expected for the model now proposed where $\Omega_{\text {total }}=\Omega_{\Lambda}+\Omega_{M}+\Omega_{A P}$.

Clouds principally of hydrogen are observed in the outer reaches of galaxies and prominently beyond galaxies, for example by radio astronomy[10]. These can be normal particles, atoms, and molecules or anti-matter. The antimatter proposed as filling the intergalactic voids may have already been detected as a portion of the gamma ray input at about $1 \mathrm{Gev}$ which is about the correct result for proton/antiproton annihilation. This model may also explain at least some of the gamma ray burst activity where apparent fireballs seem to require electron-positron and gamma ray outbursts[11]. One result that would point strongly toward this model wold be finding the annihilation energy of neutrinos to be at or near $0.000235 \mathrm{ev}$. This model may also solve the problem of how the early stages of the Universe could support both particles and antiparticles without total annihilation because of the repulsive gravitation. A further implication of this proposal: the universe has steadily expanded from the big bang but should begin to recover acceleration as the sea of antiparticles 
gradually leaves the matter portion.

John D. Anderson, et al[12] found an anomalous acceleration towards Sol of the Pioneer 10 and 11 spacecraft as they move away from Sol in opposite directions, in the Pioneer 10 case of $\mathrm{a}=8.74 \times 10^{-8} \mathrm{~cm} / \mathrm{sec}^{2}$. Assume that $\mathrm{G}$, the gravitational constant, is the same for matter and antimatter. Using $a=\mathrm{Gm} / \mathrm{r}^{2}$ where $\mathrm{r}$ is chosen a about half the radius of the universe or $5 \times 10^{9}$ light years, one finds $\mathrm{m}$ to be 4.2 $\times 10^{39} \mathrm{Kgm}$, a reasonable number, a large fraction of the mass of the universe.

It seems that Immanuel Kant had another correct intuition in proposing a repulsive force in the "heavens" [13].

For the advice of James Lindesay and Pierre Noyes I am grateful.

Received 6 June 3006

\section{Resumé}

On le constate que l'énergie foncée se compse de l'anti-matter gravitationallement repulsive. Les implicacions sont discutées. La preuve systéme solaire est fourue.

The paper by Walter Lamb reproduced above proposes a new approach to some of the problems with which cosmology is currently plagued. His model postulates a novel kind of antimatter which repels ordinary matter and also is self-repulsive. His proposal is said to be an extension of an idea that I suggested, after a conversation with Scott Starson[1]. My justification for suggesting that the force of gravity, like electrostatic force, reverses sign when one of the two particles is changed to an "antiparticle" was based on the "crossing symmetry" of relativistic quantum field theory and the CPT theorem. The asymmetry between matter and antimatter that Lamb postulates clearly violates that assumption, and hence his argument cannot invoke that justification. I will treat Lamb's suggestion as a novel proposal in what follows. For my current position on "antigravity" see[14], Ch.'s 8, 9; for Starson's views see[15].

Given an antimatter that is both self-repulsive and repels matter, Lamb anticipates that currently his antimatter will occupy the large voids which occur between clusters and strings of clusters of visible galaxies in Hubble pictures of our current 
universe, thus explaining the fact that the universe "looks like a sponge". Judging from the tremendous effort using super-computers that it takes to actually calculate something that looks like these observations[16], I am dubious that Lamb's model would actually lead to similar results if put to computational test.

The observational fact that Lamb's proposal addresses most immediately is that the very large rate of expansion of our universe from some state of very high density at (or soon after [18]) "the big bang" (about $13.7 \pm 0.2$ billion years ago) has been steadily decreasing until about 6 billion years ago when it started to increase at an ever increasing rate. Lamb anticipates that "the universe has steadily expanded from the big bang [at a decelerating rate] $]^{\S}$ but should begin to recover acceleration as the sea of antiparticles gradually leaves the matter portion." I agree that his theory does suggest that something like what is observed could be a consequence of his model; proof of this conjecture could supply a strong point in favor of Lamb's conjecture.

Lamb goes on to state that "This approach and understanding solves all or nearly all of the dark energy problem.". By the "dark energy problem" I take him to mean that, given the observational result that $\Omega_{\text {total }}=1$ and assuming that $\Omega_{\text {total }}=$ $\Omega_{\Lambda}+\Omega_{M}$ then current observations support the conclusions “... $\Omega_{\Lambda}$ (vacuum energy density $)=0.7$, and $\Omega_{M}$ (matter density $)=0.3$ ". For logical completeness $I$ would add the remarks: (1) Lamb's notation presupposes that the reference model is a flat space with energy density normalized to the Friedman-Lemaître solution of the Einstein "matter only" general relativistic (GR) cosmology at the critical density and the observed Hubble constant. (2) Since the numbers only apply to the currently observed structure of a demonstrably[17] dynamically evolving universe, Lamb also puts himself under the obligation to show what time history of the relative amounts of the various constituents he allows are predicted by his model.

Lamb then goes on to say that "Here the term vacuum energy density may be about half replaced by anti-particle dark energy, $\Omega_{A P}$. The 0.7 value is close to double the 0.3 value which is expected for the model now proposed where $\Omega_{\text {total }}=$ $\Omega_{\Lambda}+\Omega_{M}+\Omega_{A P}$." As best I can interpret what Lamb had in mind here is that his

\footnotetext{
${ }^{\S}$ Comment in [] added by HPN to bring quoted text into accord with observational "facts".
} 
novel type of antimatter would still exist in the universe and be quantitatively still equal, or nearly equal, to the amount of matter. Because it would squeeze matter and antimatter away from itself it would be the only occupant of the voids between galaxies and galactic clusters in our present universe. Further [if its annihilation cross section on encountering matter under current conditions were small enough] it would still be occupying a lot of the space where ordinary matter is observed today. Because of its repulsive action on both matter and itself, it would be supplying an energy density effect about twice as much as the energy density effect of matter as measured at present by current observations interpreted in the usual way. Then, to the extent that $0.3+0.3+0.3 \approx 1$, "all or nearly all" of the dark energy problem is solved. I find this problematic, as already noted in point (2) of the last paragraph, until the dynamics of Lamb's constituents $\Omega_{\Lambda}, \Omega_{M}$ and $\Omega_{A P}$ are either fitted into the conventional GR framework or used to construct a calculable theory that goes beyond GR and can be shown to predict unambiguously the current observational results.

For some time I was discussing all of these problems every week with Walter Lamb. I suggested to him that he submit something like the draft given above to Physics Essays so that we could get referee comments that we could work on together and develop a final version for him to submit, which - as explained in my introductory footnote to this paper - I would refuse in advance to referee. He did make the initial submission, but unfortunately illness and death prevented him from completing the task. My remarks above touch on some of the points I would have expected a referee to raise.

I might also note that Lamb's conjecture would also have to address problems like whether dark matter — which contributes about five times as much energy density to the universe as ordinary matter[19] — has a Lamb-type antimatter associated with it, what role radiation plays in his cosmological history, during what period in time dark matter and dark antimatter "freeze out" of the expanding radiation-dominated early universe, etc... Another problem is that Lamb suggests that "The antimatter proposed as filling the intergalactic voids may have already been detected as a portion of the gamma ray input at about $1 \mathrm{Gev}$ which is about the correct result for proton/antiproton annihilation." This would put quantitative constraints on his model, 
if explored. His remark "One result that would point strongly toward this model would be finding the annihilation energy of neutrinos to be at or near 0.000235 ev." would place similar constraints. It has been shown that massive neutrinos (sometimes called "warm dark matter") could easily "fuzz out" the large scale structure ("the sponge") now observed if they provide even small amounts of the mass-density at early times[17]; these problems would be compounded if Lamb's suggestion is correct.

One reason I wanted Lamb's paper to be submitted and discussed was because Lamb's attempt to explain how the space-time anomalies in the Pioneer spacecraft data might be accounted for was the first $I$ had seen that depended on a theory that had been constructed for another purpose. While preparing Lamb's paper for posthumous publication I learned (from Dr. W.H.Brill) of the paper by P.A.LaViolette which points out that he predicted the effect long ago and had been actively engaged in trying to get his prediction tested for some time[20]; I have not had time to give LaViolette's paper the attention it obviously deserves. I hope that this paper by Lamb will give positive reinforcement to LaViolette's efforts to get the establishment to put more resources into examining this effect. Much could hang on it.

After submitting this manuscript I received two referee comments which raise points that need clarification. I am indebted to the referees both for their thoughtful responses to Walter Lamb's ideas and for this opportunity to respond.

(Referee 1) “... Like (plus or minus) charges repel,...but like masses (plus or minus) attract. ... So Lamb's idea that there is self-repulsion of sub-portions of anti-matter (treated as negative masses) is hard to grasp. Two negative masses (anti-matter), like two positive masses (matter), ought to attract, not repel." This is precisely where I part company with Walter Lamb. I agree with the referee (in the context of the Noyes-Starson paper and my subsequent work) - (see[14], Ch.'s 8, 9) — as already noted above.

The referee goes on: "Still, if he [Lamb] is right, it [i.e Lamb's conjecture] might be made to fit with recent observations of an unprecedented huge void in the cosmos, said to be over a billion light years across. But I find it puzzling, because this void was detected as a cold spot on the CBR distribution. Why would a region filled with anti-matter look cold? Would not anti-matter undergo kinetic motions like those of 
matter? And would this motion not by definition be 'heat'? Maybe the IR photons emitted by kinetic motions of anti-matter convey 'cold' energy rather than hot?" Here part of this referee's puzzle could be resolved by noting that the "void" he refers to (and the "voids" Lamb refers to) are due the absence of radiating matter (stars and galaxies of stars) as observed at optical wavelengths by the Hubble space telescope. There are no voids in the CMB, which follows Planck's black body radiation curve for a temperature of about $2.73^{\circ} \mathrm{K}$ to a few parts in a hundred thousand. So, to that accuracy, space in all directions has the same temperature.

Indirectly, however, the referee has put his finger on two real problems with Lamb's model if one tries to make it consistent with observation. The first is that Lamb assumes that his antimatter is transparent to electromagnetic radiation. Following my interpretation of Lamb's taking $\Omega_{M}=0.3 \approx \Omega_{d M}$ as referring to dark matter(dM), it would be consistent to assume this. Currently dark matter is only directly observed by gravitational lensing of the energy content of electromagnetic radiation[17], which has no "rest mass". This raises a new question. Does dark antimatter(dAM) attract electromagnetic energy? If so, $\Omega_{d A M} \approx 0.3$ in the voids should be relatively easy to detect or rule out using current techniques or possibly by using currently available data. If dark antimatter(dAM) repels electromagnetic energy there would be no observable lensing effect. In either case dAM would have very different effects on the time history and the dynamics of the cosmos during the radiation-dominated era; both cases would have to be worked out and compared with existing fits to the observational data before most cosmologists would find Lamb's conjecture worth exploring. Either case clearly violates the equivalence principle and hence is incompatible with GR, which brings us to the objection raised by the second referee.

(Referee 2) "I wonder how the authors' model would confront the principle of equivalence (of gravitational and inertial mass). Gravitational mass would seem to be negative for a positron and positive for an electron. If the principle of equivalence were invoked for, say, a positron-electron annihilation (as in a PET-scan device), then I think the authors' theory would predict the transformation of a net zero mass into a huge energy - contradicting mass/energy equivalence in quite a pedestrian context. Somehow this logic should be examined in light of the theory. But the ideas are well 
enough expressed and the paper itself is such a refreshing example of dialogue that it should be published in Physics Essays."

Again, I agree with the referee, and admit that this is a valid objection to my own work which entertains the possibility of a crossing-symmetric gravitational interaction that predicts that anti-protons will "fall up" near the surface of the earth. Lamb does cite a paper by Koberlein[2] which does attempt to show how GR might be extended to include "repulsive antigravity". To quote from Koberlein's conclusion: "... It is clear, however, that matter-antimatter repulsion does not contradict current theoretical models outright. Rather it relegates them to special cases of a much richer universe." I suspect that Koberlein would have even more difficulty proving that such a statement applies to Lamb's conjecture. My advice to any young researcher who wants to take Lamb's (or my) conjecture further is that he wait for some empirical evidence of anomalous gravitational behavior by antiprotons, or has some compelling reason coming from changes in the empirical, observational or theoretical landscape that turns him in this direction.

Finally, I believe strongly that alternatives to GR need exploration for reasons of logical consistency. General relativity requires that, locally, geometrodynamics approach the "flat" space-time of special relativity which can have no preferred coordinate frame. Yet the coordinate system in which the cosmic microwave background (CMB) radiation is at rest provides the unique frame to which all cosmological models have to be referred. In fact, our own quantitatively observed motion - and that of the solar system itself - relative to that frame have sometimes been called "the new ether drift." This suggests to me that models which keep Euclidean 3-space separate from the observed Lorentz time dilation and the related particulate mass increase with velocity might in the long run lead to a simpler and more powerful cosmology. One such starting point could be provided by the "neo-Hertzian Electrodynamics" of Tom Phipps[21], an author who is certainly familiar to many readers of Physics Essays. I hope that, despite the many thorny problems it raises, Lamb's paper will, in a modest way, keep such alternative approaches to these fundamental problems alive. 


\section{References}

[1] H.Pierre Noyes and Scott Starson. "Discrete Antigravity", SLAC-PUB-5429, Mar 1991. 16pp.

[2] B.D.Koberlein, "Crossing Symmetry and the Equivalence Principle in Einsteinian Gravity", in Aspects II: Proc. ANPA 20, K.G.Bowden, ed. pp 249-255.

[3] R.J.Hughes and M.H.Holtscheiter, Phys. Rev. Letters 66, 850 (1991).

[4] E.G.Adelberger, B.R.Hecke, C.W.Stubbs and Y.Su, Phys. Rev. Letters 66, 850 (1991).

[5] J.A.Wheeler and K.Ford, Geons, Black Holes and Quantum Foam, Norton, New York, 2000, p 116.

[6] F.Hoyle, G.Burbridge, and J.V.Narliker, A Different Approach to Cosmology, Cambridge University Press, 2000, p 109.

[7] H.Kurki-Suonio and E.Sihvola, Phys. Rev. Letters 84, 3756 (2000).

[8] For example, Scientific American, May (2000), p.23.

[9] C.Seife, Science, 288, 598 (2000).

[10] M.Zwaan, Science, 288, 822-3 (2000).

[11] R.Mészáros, Science291, 79 (2001).

[12] J.Anderson, et. al., Physical Review Letters, 81, 2858 (1998).

[13] I.Kant, Universal Natural History and Theory of the Heavens (1755). For alternative theories see Martin Rees, Astrophysical Cosmology.

[14] H.Pierre Noyes, BIT-STRING PHYSICS: A Finite and Discrete Approach to Natural Philosophy, edited by J.C. van den Berg. World Scientific, Singapore (1991), 557 pp. 
[15] S.Starson, "Fundamental Units, Unification, and the Age of the Universe", in Alternatives, Keith G. Bowden,ed., 1995, pp 300-323; (Proc. ANPA 16).

[16] Robert Nichol, Observation of Large Scale Structure[17].

[17] $35^{\text {th }}$ SLAC Summer Institute, Dark Matter: from the Cosmos to the Laboratory, SLAC, Menlo Park, CA, August 2007: http:// wwwconf.slac.stanford.edu/ssi/2007/lateReg/program.htm

[18] James Lindesay, "Consequences of a Cosmological Phase Transition at the Tev Scale", Foundations of Physics (online 13 March 2007) 41 p.

[19] Scott Dodelson, Cosmology for Particle Physicists[17].

[20] P.A.LaViolette, "The Pioneer Laser Signal Anomaly: Possible Confirmation of Spontaneous Photon Blueshifting", Physics Essays, 18, 150-163 (2005).

[21] Thomas E.Phipps, Jr. Old Physics for New - a worldview alternative to Einstein's relativity theory, Aperion (C. Roy Keys Inc, 4405 rue St-Dominique, Montreal, Quebec H2W 2B2 Canada) (2006). 